\title{
$\Omega$
}

\section{New Results on Mass Measurements of Stored Neutron-Rich Nuclides in the Element Range from Pt to $\mathrm{U}$ with the FRS-ESR Facility at 360-400 MeV/u}

L. Chen ${ }^{a, b, 1}$, W. R. Plaßª,b, H. Geissel ${ }^{a, b, *}$, R. Knöbel ${ }^{b}$, C. Kozhuharov ${ }^{b}$, Yu. A. Litvinov ${ }^{b}$, Z. Patyk ${ }^{c}$, C. Scheidenberger ${ }^{a}, b, K$. Siegień-Iwaniuk ${ }^{c}$, B. Sun ${ }^{b, d}$, H. Weick ${ }^{b}$, K. Beckert ${ }^{b}$, P. Beller ${ }^{b}$, F. Bosch ${ }^{b}$, D. Boutin ${ }^{b}$, L. Caceres ${ }^{b}$, J. J. Carrolle, D. M. Cullen ${ }^{\text {f }}$, I. J. Cullen ${ }^{\text {, }}$, B. Franzke ${ }^{\text {, }}$, J. Gerl ${ }^{\mathrm{b}}$, M. Górska ${ }^{\mathrm{b}}$, G. A. Jones ${ }^{\mathrm{g}}$, A. Kishada ${ }^{\mathrm{f}}$, J. Kurcewicz ${ }^{\mathrm{b}}$, S. A. Litvinov ${ }^{\mathrm{b}}$, Z. Liu ${ }^{\mathrm{g}}$, S. Mandal ${ }^{\mathrm{b}}$, F. Montes ${ }^{\mathrm{h}}$, G. Münzenberg ${ }^{\mathrm{b}}$, F. Nolden ${ }^{\mathrm{b}}$, T. Ohtsubo ${ }^{\mathrm{i}}$, Zs. Podolyák ${ }^{\mathrm{g}}$, R. Proprie ${ }^{\mathrm{e}}$, S. Rigby ${ }^{\mathrm{f}}$, N. Saito ${ }^{\mathrm{b}}$, T. Saito ${ }^{\mathrm{b}}$, M. Shindo ${ }^{\mathrm{j}}$, M. Steck ${ }^{\mathrm{b}}$, P. M. Walker ${ }^{\mathrm{g}}$, S. Williams ${ }^{\mathrm{g}}$, M. Winkler ${ }^{\mathrm{b}}$, H.-J. Wollersheim ${ }^{\mathrm{b}}$, T. Yamaguchi ${ }^{\mathrm{k}}$

a Justus-Liebig-Universität Gießen, Heinrich-Buff-Ring 16, 35392 Gießen, Germany

${ }^{b}$ GSI Helmholtzzentrum für Schwerionenforschung, Planckstraße 1, 64291 Darmstadt, Germany

${ }^{c}$ National Centre for Nuclear Research, Hoża 69, 00-681 Warszawa, Poland ${ }^{d}$ School of Physics and Nuclear Energy Engineering, Beihang University, Beijing 100191, China

${ }^{e}$ Youngstown State University, One University Plaza, Youngstown, Ohio 44555, USA

${ }^{f}$ University of Manchester, Oxford Road, Manchester, M13 9PL, United Kingdom ${ }^{g}$ Department of Physics, University of Surrey, Guildford, GU2 7XH, United Kingdom

${ }^{h}$ Michigan State University, East Lansing, Michigan 48824, USA

${ }^{i}$ Department of Physics, Niigata University, Niigata 950-2181, Japan

${ }^{j}$ Department of Physics, University of Tokyo, Tokyo 113-0033, Japan

${ }^{k}$ Department of Physics, Saitama University, Saitama 338-8570, Japan

\begin{abstract}
Masses of ${ }^{238} \mathrm{U}$ projectile fragments have been measured with time-resolved Schottky Mass Spectrometry (SMS) at the FRS-ESR facility at GSI. The exotic nuclei were created in the production target at the entrance of the fragment separator FRS, spatially separated in flight and injected into the

\footnotetext{
*Corresponding author; electronic address: H.Geissel@gsi.de

${ }^{1}$ Present address: Cyclotron Institute, Texas A \& M University, Texas 77843, USA
} 
storage-cooler ring ESR at about $70 \%$ light velocity. This means the ions were mainly bare or carried only a few electrons, e.g., the population of Li-like ions was below $1 \%$ for Pt fragments. Accurate new mass values of 33 neutron-rich, stored exotic nuclei in the element range from platinum to uranium have been obtained for the first time. In total more than 150 nuclides including references with well-known masses have been covered in this large-area SMS measurement. A novel data analysis has been applied which reduces the systematic errors by taking into account the velocity profile of the cooler electrons and the residual ion-optical dispersion in this part of the storage ring. The experiment, the data analysis, and the mass values are presented. The experimental data are compared with theoretical predictions demonstrating systematic deviations of up to $1500 \mathrm{keV}$ from modern mass models.

Keywords: $21.10 . \mathrm{Dr}, 21.60 .-\mathrm{n}, 200<=\mathrm{A}<=240,32.10 . \mathrm{Bi}$

\section{Introduction}

Heavy neutron-rich nuclides play an essential role in nuclear structure and nuclear astrophysics. The understanding of the rapid neutron capture nucleosynthesis process (r-process) and thus the origin of the heavy elements strongly relies on the knowledge of their nuclear properties, e.g. masses and half-lives [1]. Modeling of the r-process is also required to determine the initial abundances of cosmochronometers, such as uranium and thorium, which can be used to determine the age of our galaxy [2]. In nuclear structure research, the neutron-rich regions of the $Z=82$ and $N=126$ shell closures are of particular interest. For example, they are needed to understand and predict the stability of superheavy elements [3]. Despite their importance, our knowledge about neutron-rich nuclides above lead is still rather scarce because their production cross sections are very small and the separation and particle-identification techniques for these heavy ions are very difficult [4].

The goal of the present experiment, performed in 2004, was to extend the knowledge of the outskirts of neutron-rich nuclides between lead and uranium. Uranium projectile fragments were measured at the FRS-ESR facility $[5,6,7]$ using time-resolved Schottky Mass Spectrometry (SMS) [8, 9, 10]. The results of this experiment include the discovery of neutron-rich isotopes, isomers, and the determination of new mass values and half-lives. Some 


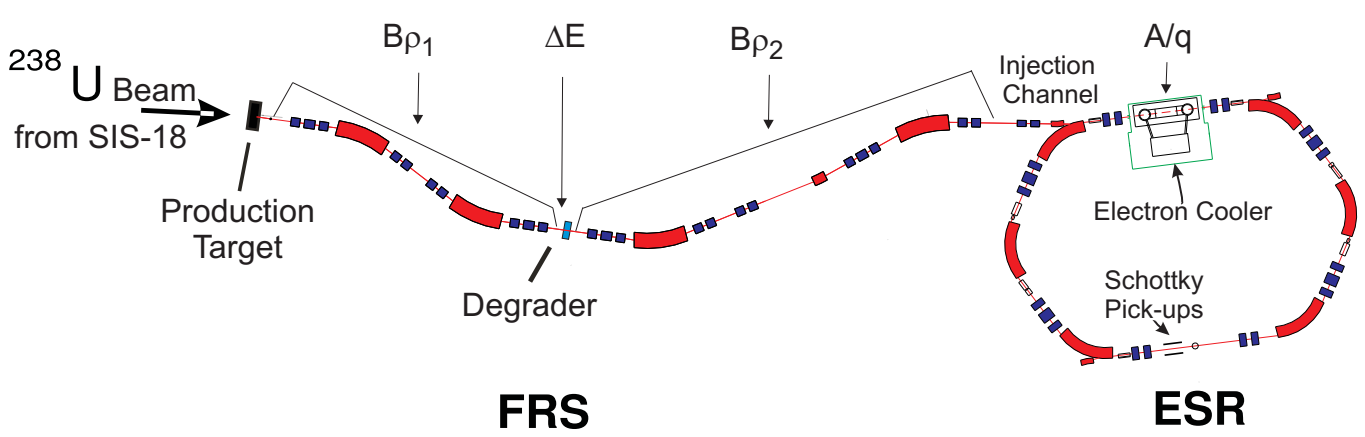

Figure 1: Experimental setup employed for the mass measurements. The fragments of interest were spatially separated in flight with the FRS applying a two-fold magnetic rigidity analysis combined with atomic energy loss in degraders, the $\mathrm{B} \rho-\Delta \mathrm{E}-\mathrm{B} \rho$ method. Additional separation and identification criteria were provided by the narrow $\mathrm{B} \rho$ acceptance window of the ESR entrance $(\leq \pm 0.2 \%)$ and the electron cooling, which enforces that all stored nuclei circulate with the same mean velocity.

of the results of this experiment have already been published in references $[11,12,13,14,15]$. The purpose of this paper is to present the values of the mass surface of neutron-rich nuclides between platinum and uranium deduced by a novel analysis method.

Since the experiment has been performed, new mass values obtained by Penning trap measurements have been published on the masses of some of the same nuclei $[16,17,18]$. This allows to make a comparison between the two accurate methods of SMS [7, 8] and Penning trap [19, 20, 21] in this new area of experimental masses. Furthermore, recently a breakthrough has been achieved in nuclear structure studies with ${ }^{208} \mathrm{~Pb}$ and ${ }^{238} \mathrm{U}$ projectile fragments at relativistic energies $(1000 \mathrm{MeV} / \mathrm{u})$ [22, 23, 24] with the separator FRS [5], reaching yet more neutron-rich nuclei.

\section{Experiment}

In this experiment a $670 \mathrm{MeV} / \mathrm{u}{ }^{238} \mathrm{U}$ projectile beam was extracted from the heavy-ion synchrotron SIS [25] with a maximum intensity of $2 \times 10^{9} /$ spill and focused on a $4 \mathrm{~g} / \mathrm{cm}^{2}$ beryllium production target placed at the entrance of the fragment separator FRS. Fast extraction was used with a spill length of $300 \mathrm{~ns}$ and a typical repetition rate of 0.2 per minute. The fragments of interest were separated in flight with the FRS and injected into the storagecooler ring ESR [6] (Fig. 1). The main goal of this experiment was to perform 
mass measurements. Therefore, the separation conditions at the FRS were selected such that, in addition to the nuclei of interest, sufficient reference nuclides were injected into the ESR and recorded in the same revolutionfrequency spectra. Two-fold magnetic rigidity analysis combined with atomic energy loss in a very thin degrader, with a thickness of $50 \mathrm{mg} / \mathrm{cm}^{2}$ (plastic), was selected to restrict the transmitted range of elements between gold and uranium. Some of the measurements were also performed with pure magnetic rigidity separation of the FRS. The latter and simpler separation method can be applied, if the intensities of the different ions injected into the storage ring are sufficiently low such that correlations of the circulating ions due to Coulomb interaction are excluded and the individual signals in the revolution frequency spectrum can be well resolved [8].

Electron cooling was applied to the ion beam stored in the ESR. This forces the circulating ions to the same mean velocity, which is determined by the chosen terminal voltage of the electron cooler. In the present experiment, the velocity of the ions was about $70 \%$ of the velocity of light, corresponding to kinetic energies in the range of 360 to $400 \mathrm{MeV} / \mathrm{u}$. After electron cooling, the velocity spread of low intensity stored fragments amounts to approximately $5 \times 10^{-7}[7,8,26,27]$. The revolution frequencies of two different cooled ions $i$ and $j$ are then related by

$$
\frac{f_{i}-f_{j}}{f_{i}}=-\alpha_{p}\left[\frac{(m / q)_{i}-(m / q)_{j}}{(m / q)_{i}}\right]+\left(1-\frac{\gamma^{2}}{\gamma_{t}^{2}}\right)\left(\frac{v_{i}-v_{j}}{v_{i}}\right)
$$

where $\alpha_{p}$ is the momentum compaction factor which is defined by

$$
\alpha_{p}=\frac{d C / C}{d(B \rho) /(B \rho)}
$$

with $C$ representing the orbital length of the stored ions and $B \rho$ the magnetic rigidity. The momentum compaction factor $\alpha_{p}$ has been determined over the full $m / q$ range of the storage acceptance of the ESR. In this way, each peak in the frequency spectrum corresponds to a specific mass-to-charge value. The parameter $\gamma_{t}$ corresponds to the transition energy characteristic for the ion-optical setting of the storage-ring and is given by the relation $\gamma_{t}=\frac{1}{\sqrt{\alpha_{p}}}$.

After injection of the ions into the ESR, only a part of the full storage momentum acceptance $( \pm 1.25 \%)$ is filled, since the injection acceptance is much smaller $( \pm 0.2 \%)$ than the storage acceptance. Due to atomic collisions electron cooling forces all stored ions to the velocity of the electrons. This 
disperses their different mass-to-charge ratios, such that only a part of the cooled ion species remains within the storage acceptance. In order to access a larger mass-to-charge range, different settings of the cooler voltages were used. Since the cooling time is proportional to the third power of the velocity difference between the cooler electrons and the circulating fragments [28], a scan of the cooler voltage also leads to a shorter cooling time for the selected fragments and hence access to shorter-lived nuclei. The cooler voltage was changed in steps of $2 \mathrm{kV}$ in the range from $190 \mathrm{kV}$ to $200 \mathrm{kV}$, corresponding to mass-to-charge ratios from 2.609 to $2.682 \mathrm{u}$ /elementary-charge. Cooler currents between 200 and $475 \mathrm{~mA}$ were used.

For the measurement of the revolution frequency spectra, the current signals induced by the circulating few-electron heavy ions at each revolution on two metallic pick-up plates in dipolar arrangement were recorded. The signal of the pick-up plates, e.g., from the $31^{\text {st }}$ harmonic, was tuned in a resonance circuit, amplified, summed and shifted down by a frequency of about $59 \mathrm{MHz}$, which was provided by a local oscillator (LO), to the frequency range of $320 \mathrm{kHz}$ using an image-reject mixer. The resulting signal was split into two parts, one for on-line monitoring and one for off-line analysis. The latter part was digitized using an ADC with $640 \mathrm{kSamples} / \mathrm{s}$ and written to the data storage system in blocks with a length of about $0.1 \mathrm{~s}$.

\section{Analysis}

\subsection{Generation of Frequency Spectra}

The frequency spectra of the stored ions were generated by Fast Fourier Transform (FFT). Two blocks of data were combined to time domain sections with a length of about $0.2 \mathrm{~s}$ each. Every FFT spectrum contained 65536 channels with a channel width of about $4.8 \mathrm{~Hz}$. To achieve an optimum signal-to-noise ratio, 100 of such individual FFT spectra were averaged to a single revolution frequency spectrum, which thus corresponds to $20 \mathrm{~s}$ of data.

A typical frequency spectrum is shown in Fig. 2. SMS shares the advantage of other broadband mass spectrometry methods, such as time-of-flight mass spectrometry, of being able to simultaneously observe many different ion species, including nuclides with known masses and nuclides with hitherto unknown masses. This gives SMS an exceptionally high sensitivity. Note that, in addition, in the element range of the present experiment, SMS can detect single ions [8], and that a single detected ion is sufficient to obtain 

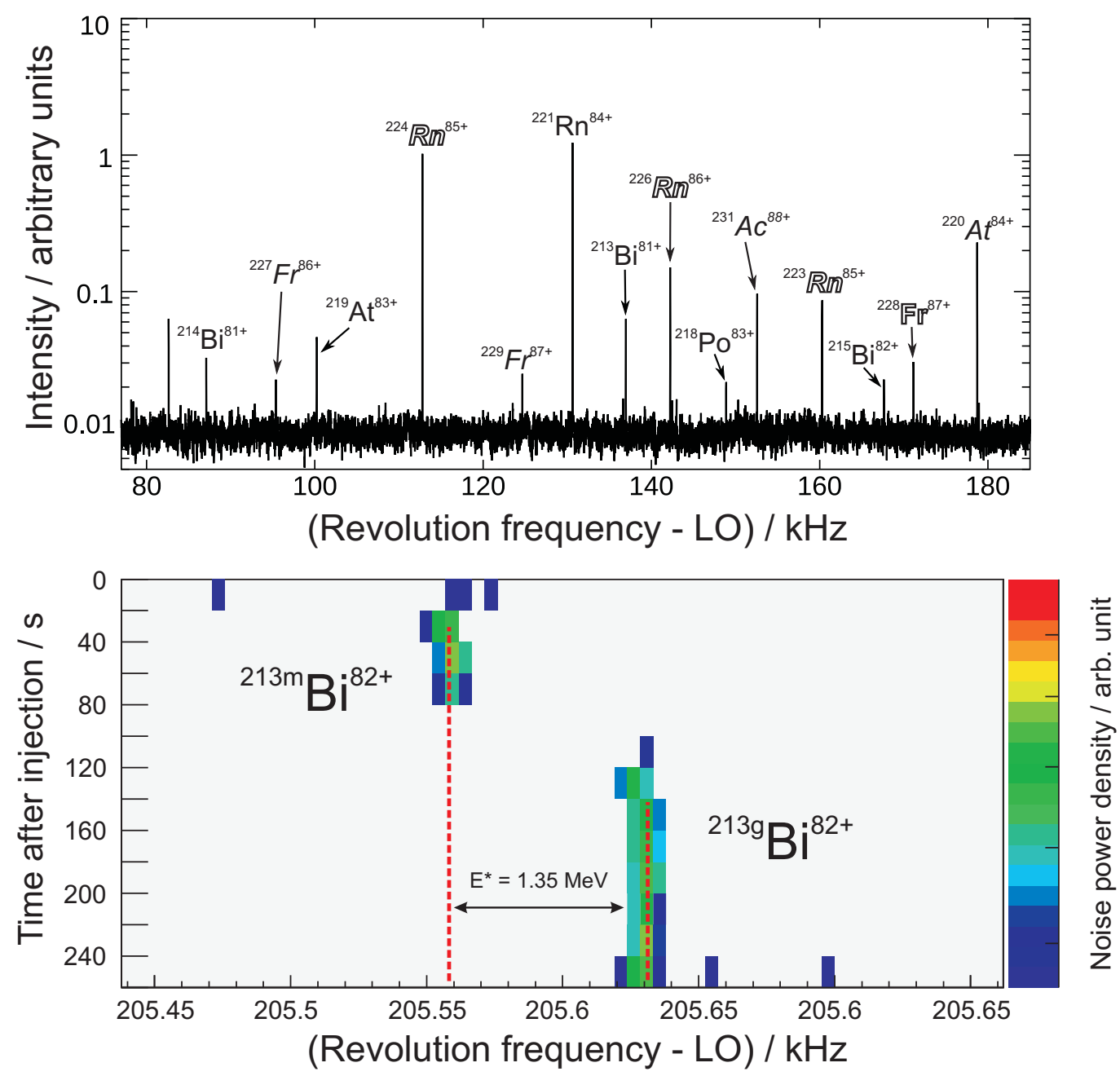

Figure 2: Upper panel: Characteristic Schottky frequency spectrum with isotopes of wellknown masses and those with unknown masses indicated by outlined element names. Note, the frequency range in the plot results from the subtraction of a fixed oscillator frequency (LO) which amounts to about $59 \mathrm{MHz}$. Lower panel: Example of a single-particle spectrum for the new isomer ${ }^{213 m} \mathrm{Bi}$ and its decay to the ground state. The recording conditions were slightly different compared to the large-range spectrum above as indicated by the different frequencies and charge states for ${ }^{213} \mathrm{Bi}$ ions in both spectra. The excitation energy of the isomeric state has been determined to $(1.353 \pm 0.021) \mathrm{MeV}$.

a mass value as shown in the lower panel of Fig. 2, where a single ${ }^{213} \mathrm{Bi}$ ion being firstly in the isomeric state and then undergoing $\gamma$-decay into its 
ground-state was discovered in the time resolved spectrum. The discussion of further isomers discovered in this experiment will be the subject of a forthcoming publication.

Also, the broadband capabilities of SMS allow for internal calibration of the spectra. In particular, frequency drifts caused, e.g. by instabilities of the power supplies for the ESR magnets, can be easily corrected for, as will be discussed below. Thus, a much higher resolving power can be achieved than could be expected from the stabilities of the magnetic fields. The measured mass resolution was about $6 \times 10^{-7}$. Hence, isobars and even isomers with excitation energies of down to about $0.1 \mathrm{MeV}$ could be resolved.

For each injection, 13 of such Schottky spectra were generated with their corresponding time interval of $20 \mathrm{~s}$. The signal of the injection kicker magnet started the measurement cycle of about $260 \mathrm{~s}$, see lower panel of fig. 2 . With this time information the lifetime of the stored exotic nuclei can also be measured, if the decay happens during the recording period. The timeresolved measurement is also important for proper identification of some of the frequency peaks observed, e.g. in the case of low-lying isomers. In total, 8177 spectra were analyzed.

\subsection{Analysis of Schottky Frequency Spectra}

As in previous SMS experiments [8] a coupled motion of the circulating ions with close mass-to-charge ratios was observed. It can be explained by the Coulomb interaction of the ions, which precludes overtaking of the heavier by the lighter ion [29]. If the mass-to-charge difference and therefore the radial distance of the ions is large enough, the nuclides follow an independent trajectory resulting in well resolved frequencies. Cases of coupled motion of charged ions were excluded in the analysis. This can readily be done since the signals of two coupled ions appear as a single mixture peak in the frequency spectrum, which has a significantly larger width and whose frequency shows a random jitter.

Next, the peaks were fitted with a Gaussian distribution to determine the mean revolution frequencies. Due to instabilities of the power supplies for the ESR magnets or the cooler voltage, the mean revolution frequencies show drifts of up to a few hundred Hertz (at the $31^{\text {st }}$ harmonic) over a period of several hours $[8,10]$. However, since all ions are subject to the same frequency drifts, these drifts can be corrected for by shifting all peaks in an individual spectrum by the same amount. All spectra belonging to one experimental setting, i.e. all spectra that have been acquired with the same electron cooler 
voltage and current, can then be combined in a single projected spectrum for identification of the nuclides. In order to determine the correction value for every spectrum, for each experimental setting the spectrum containing the maximum number of peaks was first chosen as a pattern spectrum. Next, the mean revolution frequencies of the other spectra belonging to the same setting were shifted such that the best match between the pattern spectrum and each other spectrum was obtained. After application of these shifts, a projection spectrum was created for each setting by summing the mean revolution frequency peaks of all spectra. The width of the peaks in the projection spectra amounts to about $3 \mathrm{~Hz}$ (FWHM).

\subsection{Identification of Peaks}

The identification of the peaks was performed using a pattern recognition algorithm [27]. The best match between the projection spectrum and a theoretical spectrum was calculated for each setting, yielding the identification of each experimentally observed peak. The theoretical spectrum was created from a list of nuclides that can be produced by fragmentation or fission of relativistic uranium ions. Nuclide data was taken from the Atomic Mass Evaluation 2003 (AME2003) [30] and, in those cases where no mass values were available, theoretical predictions from the FRDM model [31] were considered. Charge states corresponding to 0 up to 5 bound electrons were taken into account. However, most of the ions were bare, H-like, or He-like.

The masses of these nuclides were corrected for the masses of the missing electrons and the electron binding energies [32]. Since the cooling time of several seconds does not allow to access short-lived nuclei, nuclides with half-lives in the millisecond domain could be excluded. Furthermore, the magnetic rigidity selection with the FRS and the ESR acceptance restricts the mass-to-charge range of possible candidates.

\subsection{Mass Evaluation}

Before the correlation matrix method for the derivation of the mass values for each nuclide could be applied, additional steps in the evaluation of the measured frequency spectra had to be accomplished. During the experiment the Schottky spectra were obtained under different conditions mainly characterized by the choice of the experimental parameters. Several steps had to be performed for each set of $U_{\text {Cool }}, I_{\text {Cool }}$ and the local oscillator frequency (LO) to attain the projection spectra which are used as input-data for the correlation matrix. Therefore, a spectrum with the maximum number of revolution 
times measured for one setting was taken as the pattern spectrum. Each revolution time in the pattern spectrum was assigned by $T_{i}$. The revolution times $t_{i}$ in each remaining spectrum $j$, which was obtained under the same conditions, were shifted by a constant $S_{j}$ to lead to a maximum overlap with the pattern spectrum and to minimize therewith the function

$$
l\left(S_{j}\right)=\frac{\sum_{i}^{n}\left(T_{i}-t_{i}-S_{j}\right)^{2}}{n} .
$$

where $n$ is the number of peaks being identified in both spectra. For the further analysis with the correlation matrix method only those spectra were taken into account where at least more than $33 \%$ of the peaks have been in common with the pattern spectrum and where the condition of $\sqrt{l\left(S_{j}\right)}<$ $20 \cdot 10^{-6} \mathrm{~ns}$ was fulfilled. The latter condition is approximately equivalent to $40 \mathrm{keV}$ for an ion with a charge of $\mathrm{q}=70$. About $84 \%$ of the spectra could satisfy these constraints. Finally, one projected spectrum has been obtained where the revolution time of each peak equals the average value of the revolution time obtained from the shifted revolution times. Although the pattern spectrum contains most of all measured revolution times, it does not contain all peaks found in every spectrum. Therefore, as the last step and for consistency purposes, the projected spectrum replaced the pattern spectrum and the procedure mentioned above was repeated again. After the last step one final projected spectrum for each chosen set of experimental conditions was obtained and dealt as input-data for the calculation of the mass values with the correlation matrix method $[8,27]$ which belongs to the group of the maximum likelihood methods. Since this method has been established in the analysis of data which were obtained in Schottky Mass Spectrometry (SMS) over the last years, only a short outline of the basic properties of this method shall be given here. A more detailed description can be found in [8, 27].

The likelihood function $L$ is constructed as the product of the experimental and the calibration part. We assume that each part is a product of Gaussian distributions $f(x-\mu, \sigma)$ defined by

$$
f(x-\mu, \sigma)=\frac{1}{\sqrt{2 \pi} \sigma} \exp \left(-\frac{(x-\mu)^{2}}{2 \sigma^{2}}\right),
$$

with $\mu$ as the mean value and $\sigma$ as the width of the distribution.

The calibration part $L_{c}$ is defined as

$$
L_{c}=\prod_{i} f\left(M_{i}^{b}-\widetilde{M_{i}^{b}}, \delta \widetilde{M_{i}^{b}}\right)
$$


Table 1: List of reference nuclides. Their mass values and their mass uncertainties were taken from the AME2003 compilation [30]. The mass uncertainties in keV are here given in parentheses.

\begin{tabular}{|c|c|c|c|}
\hline Isotope & Isotope & Isotope & Isotope \\
\hline${ }^{191} \operatorname{Re}(10)$ & ${ }^{207} \mathrm{~Pb}(1)$ & ${ }^{219} \mathrm{At}(4)$ & ${ }^{223} \mathrm{Ac}(7)$ \\
\hline${ }^{192}$ Os $(3)$ & ${ }^{208} \mathrm{~Pb}(1)$ & ${ }^{219} \operatorname{Rn}(3)$ & ${ }^{227} \mathrm{Ac}(2)$ \\
\hline${ }^{198} \mathrm{Pt}(3)$ & ${ }^{209} \mathrm{~Pb}(2)$ & ${ }^{220} \mathrm{Rn}(2)$ & ${ }^{229} \mathrm{Th}(3)$ \\
\hline${ }^{199} \mathrm{Pt}(3)$ & ${ }^{210} \mathrm{~Pb}(2)$ & ${ }^{221} \operatorname{Rn}(6)$ & ${ }^{231} \mathrm{Th}(2)$ \\
\hline${ }^{201} \mathrm{Au}(3)$ & ${ }^{211} \mathrm{~Pb}(3)$ & ${ }^{221} \operatorname{Fr}(5)$ & ${ }^{232} \mathrm{Th}(2)$ \\
\hline${ }^{203} \mathrm{Au}(3)$ & ${ }^{213} \mathrm{~Pb}(8)$ & ${ }^{223} \operatorname{Fr}(2)$ & ${ }^{233} \mathrm{Th}(2)$ \\
\hline${ }^{205} \mathrm{Hg}$ (4) & ${ }^{214} \mathrm{~Pb}(2)$ & ${ }^{222} \mathrm{Ra}(5)$ & ${ }^{234} \mathrm{Th}(3)$ \\
\hline${ }^{206} \mathrm{Hg}(20)$ & ${ }^{206} \mathrm{Bi}(8)$ & ${ }^{223} \mathrm{Ra}(3)$ & ${ }^{233} \mathrm{~Pa}(2)$ \\
\hline${ }^{202} \mathrm{Tl}(15)$ & ${ }^{207} \mathrm{Bi}(2)$ & ${ }^{224} \mathrm{Ra}(2)$ & ${ }^{234} \mathrm{U}(2)$ \\
\hline${ }^{203} \mathrm{Tl}(1)$ & ${ }^{208} \mathrm{Bi}(2)$ & ${ }^{225} \mathrm{Ra}(3)$ & ${ }^{235} \mathrm{U}(2)$ \\
\hline${ }^{207} \mathrm{Tl}(5)$ & ${ }^{209} \mathrm{Bi}(1)$ & ${ }^{226} \mathrm{Ra}(2)$ & ${ }^{236} \mathrm{U}(2)$ \\
\hline${ }^{208} \mathrm{Tl}(2)$ & ${ }^{211} \mathrm{Bi}(6)$ & ${ }^{227} \mathrm{Ra}(2)$ & ${ }^{237} \mathrm{U}(2)$ \\
\hline${ }^{209} \mathrm{Tl}(8)$ & ${ }^{216} \mathrm{Bi}(11)$ & ${ }^{228} \mathrm{Ra}(2)$ & ${ }^{238} \mathrm{U}(2)$ \\
\hline${ }^{210} \mathrm{Tl}(12)$ & ${ }^{210} \mathrm{Po}(1)$ & ${ }^{229} \mathrm{Ra}$ (19) & \\
\hline${ }^{206} \mathrm{~Pb}(1)$ & ${ }^{218} \mathrm{Po}(2)$ & ${ }^{230} \mathrm{Ra}(12)$ & \\
\hline
\end{tabular}

and contains the nuclear masses of bare ions $M_{i}^{b}$ as free parameters. The 58 reference masses $\widetilde{M_{i}^{b}}$ with an uncertainty of $\delta \widetilde{M}_{i}^{b}$ are taken from the AME2003 compilation [30]. Note that in the AME2003 compilation the corresponding results are always presented for neutral atoms. Therefore, we had to adapt the values to our experimental condition, i.e., taking into account the electron rest masses and the electron binding energies [8,32]. The employed reference nuclides for the present evaluation set are tabulated in Tab. 1. A mass uncertainty $\delta \widetilde{M}_{i}^{b} \leq 20 \mathrm{keV}$ was used as a criterion for selecting candidates as reference masses.

The experimental part $L_{\text {exp }}$ has the form

$$
L_{e x p}=\prod_{j, \mu} f\left(M_{j} / q_{j}-P_{n}^{\mu}\left(t_{j}\right), \delta_{j}^{\mu}\right)
$$

where the product runs over all revolution times $t_{j}$ belonging to the projected spectrum labelled by the index $\mu$. 
As already described, each ion with the revolution time $t_{j}$ in the spectra could unambiguously be identified and therewith be uniquely characterized by its number of protons $Z_{j}$, number of neutrons $N_{j}$, and the charge $q_{j}$. The mass $M_{j}$ equals to the sum of unknown bare mass values $M_{k}^{b}$ enumerated by the index $\mathrm{k}$, the mass of the orbital electrons and their (negative) binding energy $B\left(Z_{j}, q_{j}\right)[32]$ :

$$
M_{j}=M_{k}^{b}+\left(Z_{j}-q_{j}\right) m_{e}+B\left(Z_{j}, q_{j}\right) .
$$

The index k enumerates uniquely each nucleus present in the evaluated spectra.

The calibration curve in a spectrum denoted as $\mu$ is approximated by the polynomial $P_{n}^{\mu}(t)$ function of the order $\mathrm{n}$ with $\mathrm{n}+1$ unknown coefficients $a_{n}^{m}$. The width $\delta_{j}^{\mu}$ depends on the time uncertainty $\delta t_{j}$ and fulfills the relation

$$
\left(\delta_{j}^{\mu}\right)^{2}=\left(\delta t_{j} \times\left.\frac{d P_{n}^{\mu}(t)}{d t}\right|_{t=t_{j}}\right)^{2}+F^{2},
$$

with the unknown parameter F.

The masses of bare ions $M_{i}^{b}$, the coefficients $a_{n}^{m}$ of the calibration polynomials $P_{n}^{\mu}(t)$ and the free parameter $\mathrm{F}$ were treated as free parameters. Their estimated values maximize the total likelihood function $L=L_{\text {exp }} \times L_{c}$. The $\mathrm{F}$ parameter has been found iteratively to be equal to $0.233 \mathrm{keV} / \mathrm{q}$, which is equivalent to the mass uncertainty of $\delta m \approx 16.3 \mathrm{keV}$ for ions in the vicinity of $q=70$.

The statistical error has been estimated for each nuclide from the square root of the diagonal element of the inverse correlation matrix

$$
\sigma_{i}=\sqrt{\left(W^{-1}\right)_{i, i}}
$$

Elements of the correlation matrix $W$ are given by the expression (for details see Eqs. 25-26 published in the paper [27]):

$$
W_{i, j}=-\frac{\partial^{2} \ln L}{\partial M_{i}^{b} \partial M_{j}^{b}} .
$$

For each reference nuclide listed in Tab. 1 its mass and statistical error have been determined in a separate evaluation. However, in each of these evaluation loops the selected nuclide was not used as a reference. The therewith recalculated mass excess values for the reference nuclides and the list of 
newly derived mass excess values of 24 non-reference masses were the base for the estimation of the systematic error. Only nuclides with an uncertainty less than $50 \mathrm{keV}$ in the AME2003 compilation [30] and a calculated deviation to the therein given mass excess value $\left|M E_{A M E 03}-M E_{S M S}\right|<100 \mathrm{keV}$ to avoid low-lying isomers were chosen as candidates. In this way, the systematic error $\left(\sigma_{\text {syst }}\right)$ has been derived. In this analysis a value for $\sigma_{\text {syst }}=10 \mathrm{keV}$ has been deduced for $N_{n}=82$ nuclei (including all reference nuclides) solving the following equation:

$$
\sum_{i} \frac{\left(M_{i}^{b}-\widetilde{M}_{i, s y s t}^{b}\right)^{2}}{\left(\delta \widetilde{M}_{i, s y s t}^{b}\right)^{2}+\left(\sigma_{i}^{s t a t}\right)^{2}+\left(\sigma^{s y s t}\right)^{2}}=N_{n}
$$

where $\widetilde{M}_{i, s y s t}^{b}$ is the mass value and $\delta \widetilde{M}_{i, s y s t}^{b}$ the uncertainty of the nuclides chosen to provide a reliable systematic error estimation over the large Zregion covered in this experiment.

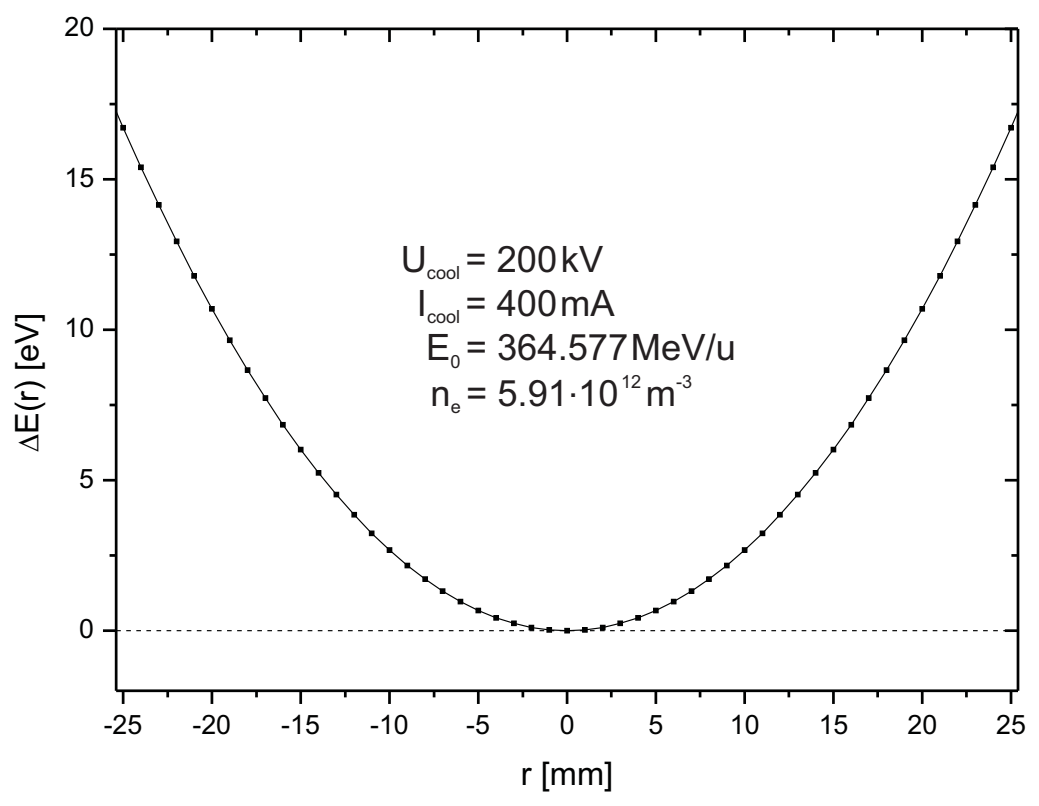

Figure 3: Calculated energy profile of the cooler electrons depending on their radial position. This energy profile results from space charge effects [28] and is additive to the chosen electron cooler energy $E_{0}$, corresponding to the terminal voltage $\mathrm{U}_{\text {cool }}$ and the current $\mathrm{I}_{\text {cool }}$. The radial dimension in the plot represents the expected electron beam size $[26]$. 
After the first evaluation process with the correlation matrix a remaining systematic dependency on the atomic number of the different nuclides was observed. These deviations are clearly manifested for the accurately known reference masses and are presented in the upper panel of figure 4 . The difference of the mass values for the reference masses in the literature [30] and the deduced corresponding values from the correlation matrix as a function of the element number demonstrates a clear correlation illustrated by the graph of a linear fit. The slope of the fitted curve is $-4.8 \mathrm{keV} / \mathrm{Z}$. This observation was not obvious in our earlier data due to a larger overall error. As a consequence, we have here investigated experimental features which could influence the accurate data in such a systematic way. A possible contribution from atomic collisions with the residual gas atoms at the ultra-high vacuum condition of $10^{-11}$ mbar in the ring and an electromagnetic interaction with the capacity probes are of minor importance in this respect. However, an explanation for this new systematic observation can be that due to the remaining ionoptical dispersion in the cooler section, the circulating ions probe the spatial velocity profile of the cooler electrons, see fig. 3. This means, the ions will have slightly different mean velocities depending where they merge with the stream of cooler electrons. This interaction directly leads to the conclusion that the second term in equation 1 cannot be neglected. In the following we demonstrate this influence quantitatively.

As stated in reference [28], the energy of the electron beam in the cooler shows a dependence on its radius which leads to a corresponding velocity shift of the stored merged ions. This shift in electron energy as a function of the radial position is given by:

$$
\Delta E(r)=n_{e} \pi r_{e} m_{e} c^{2} r^{2}
$$

where $n_{e}$ is the electron-density, $r_{e}$ the classical electron radius, $c$ the speed of light in vacuum, and $r$ the radial distance from the electron beam axis. The radial distance of the electron beam has its maximum value at about $25.4 \mathrm{~mm}$ [26] in the ESR. Figure 3 shows the calculated energy shift which is superimposed on the chosen electron cooler energy $E_{0}$. This distribution was calculated for $U_{\text {Cool }}=200 \mathrm{kV}, I_{\text {Cool }}=400 \mathrm{~mA}, E_{0}=$ $364.577 \mathrm{MeV} / u$, and $n_{e}=5.91 \cdot 10^{12} \mathrm{~m}^{-3}$ which were the conditions for one of the chosen experimental settings in the measurements presented here. The electron-density was calculated by: 


$$
n_{e}=\frac{I_{\text {Cool }}}{e \cdot v \cdot \pi r_{0}^{2}}
$$

where $I_{\text {Cool }}$ represents the cooler current, $e$ the elementary charge, $v$ the mean velocity of the electrons, and $r_{0}$ the maximum extension of the electron beam.

Theoretically, the ion-optical dispersion should be close to zero in the cooler section, however, practically it was determined to be about $1 \mathrm{~cm} / \%$ [33]. This means a momentum change of $1 \%$ causes a deflection of $1 \mathrm{~cm}$ in transverse direction. We have found similar ion-optical deviations from the calculations in recent measurements in the investigation of the isochronous mode of the ESR. For our goal to explain the observed systematic shift in the calculated mass values we have selected the reference isotopes of each element circulating in the storage ring under identical experimental conditions and determined their $\mathrm{B} \rho$ difference including the velocity profile in the electron beam of the cooler. We took into account the different cooler voltages and currents. The actual dispersion coefficient and the absolute position (offset) of the ions in the cooler are experimentally unknown at the first place and therefore, we varied both to check if the slope and the absolute values of the comparison match. Indeed, with a dispersion coefficient of $1.06 \mathrm{~cm} / \%$ and an offset of $2.4 \mathrm{~mm}$ the contribution of the radial dependence of the longitudinal energy shift $\Delta E(r)$ does explain the observed trend in the mass evaluation depicted in the upper panel of figure 4 . The result is shown in the lower panel of fig. 4 demonstrating the influence of the radial velocity profile of the cooler electrons in accurate mass measurements.

This study clearly shows that the velocity-dependent term in equation 1 has to be included in the final analysis of measured masses with the SMS method. The magnitude of this term is determined by the actual ion-optical dispersion and the characteristics of the electron cooler, i.e., the radial energy profile of the electron beam.

Although only reference nuclides, compared to the values given in the AME2003 compilation, were taken for the demonstration of this experimental influence of the velocity profile of the cooler electrons, this systematic dependence was corrected in the analysis for all mass values presented in this paper. Practically, this new observation was taken into account for the measured data by a global polynomial fit as a function of $\mathrm{Z}$, i.e. removing the observed dependence before the final loop of the evaluation with the correlation matrix was applied. As a major final result, the systematic error 

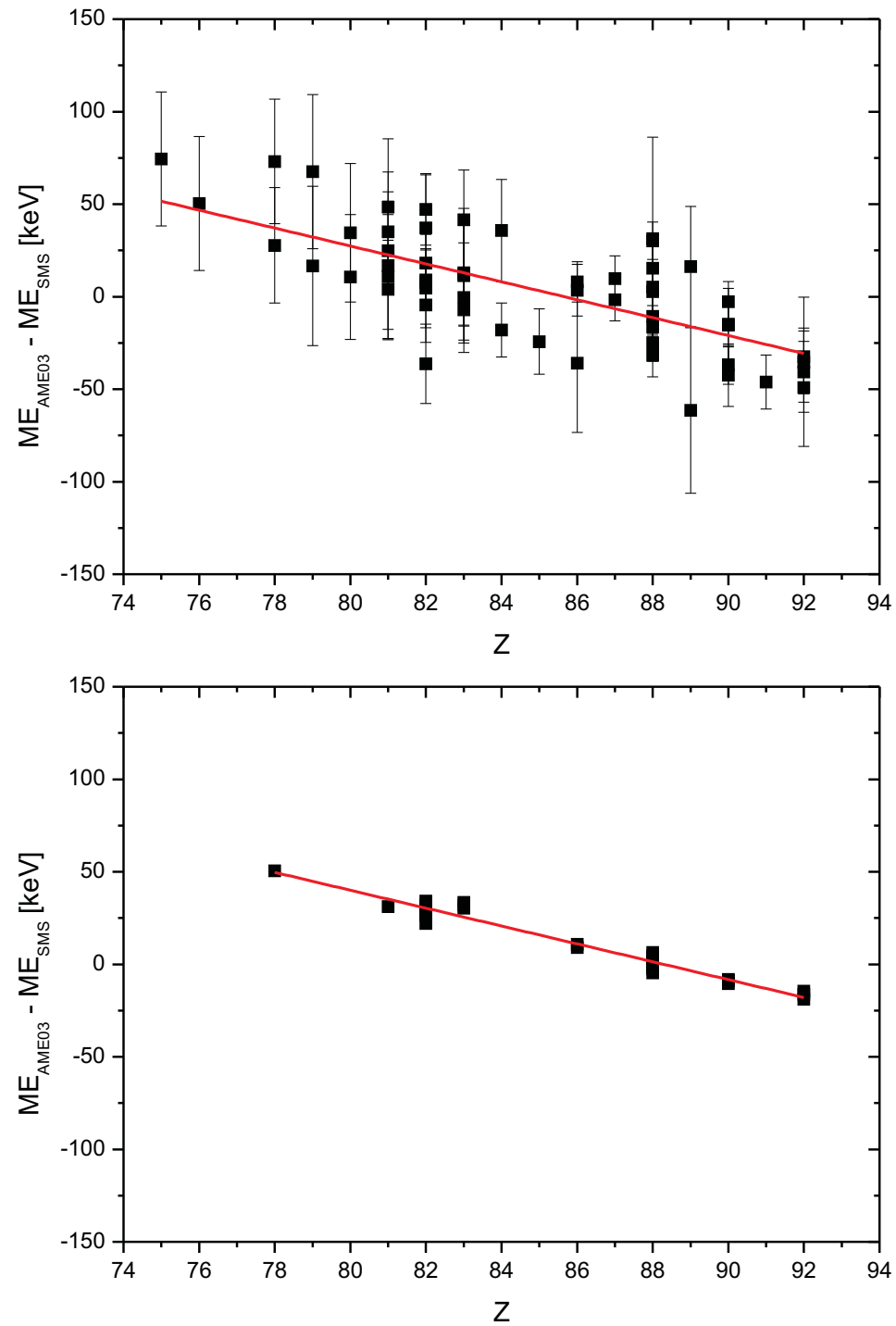

Figure 4: Upper panel: Observation of systematic deviations of reference masses to their values given in AME03 [30] found after the first evaluation with the correlation matrix method. Lower panel: Calculated deviations from the reference mass values in Atomic Mass Evaluation [30] taking into account the radial profile of the longitudinal velocity of the cooler electrons, see text. One can reproduce the observed dependence presented in the upper panel by calculations including an ion-optical dispersion coefficient of $1.06 \mathrm{~cm} / \%$ and an offset from the ion-optical axis of $2.4 \mathrm{~mm}$. 
given by equation 11 has been reduced by a factor of almost 3 compared to our previous data [8]. The final evaluated mass values are presented in the tables and figures in the upcoming sections.

Note, that the mass evaluation method used in this paper differs from the local calibration-evaluation method applied in ref. [10]. However, the mass values of both evaluation methods are in excellent agreement with an average deviation of $5 \mathrm{keV}$ and an rms value of $34 \mathrm{keV}$. The main differences are reflected in the error assignment.

\section{Experimental Results}

An overview of the mass surface covered in the experiment is given in Fig. 5. In total, more than 150 nuclides were identified in this work. Because of the high efficiency and selectivity of the in-flight separation and the performance of the time-resolved SMS the previously unknown isotopes ${ }^{213} \mathrm{Tl}$, ${ }^{221} \mathrm{Po},{ }^{222} \mathrm{Po},{ }^{224} \mathrm{At}$, and ${ }^{236} \mathrm{Ac}$ were observed [15].

The access to nuclides with the same neutron number as the primary beam can be attributed to the contribution from cold fragmentation reactions [34], e.g. ${ }^{234} \mathrm{Ra}$ resulted from a removal of 4 protons while the excitation energy is still low enough that no neutrons were evaporated. Fig. 5 also includes isotopes that were formed in nuclear charge-exchange reactions. Three very neutron-rich nuclei, ${ }^{238} \mathrm{~Pa},{ }^{237} \mathrm{Th}$, and ${ }^{236} \mathrm{Ac}$, are formed via the $(\mathrm{n}, \mathrm{p})$ reaction channel. This reaction type has also been observed with ${ }^{208} \mathrm{~Pb}$ projectiles at $1000 \mathrm{MeV} / \mathrm{u}$ in other FRS experiments [35].

For 33 nuclides the ground state mass was determined for the first time in this experiment [10]. For 31 of these nuclides, a mass value is reported in this paper. The spectra containing the remaining two nuclides, ${ }^{208} \mathrm{Hg}$ and ${ }^{211} \mathrm{Tl}$, did not fulfill the selection criteria given in Section 3.4 and thus were not included in this mass analysis. Since the local calibration-evaluation method [10] is in very good agreement with the mass values obtained with the improved correlation matrix method, the mass values for both nuclei published in $[10,14]$ are still valid.

In addition, for 22 nuclides the mass uncertainties could be reduced compared to AME2003. The mass excess $(M E)$ values obtained for the first time and the improved mass excess values are given in Table 3 . The values are given as mass excess

$$
M E=M-A \times u
$$


$\square$ isotopes with well-known masses

- known isotopes, mass values improved

$\triangle$ known isotopes, first mass measurements

$\star$ new isotopes, first mass measurements

$\triangle$ known isotopes, new mass measurements

ISOLTRAP
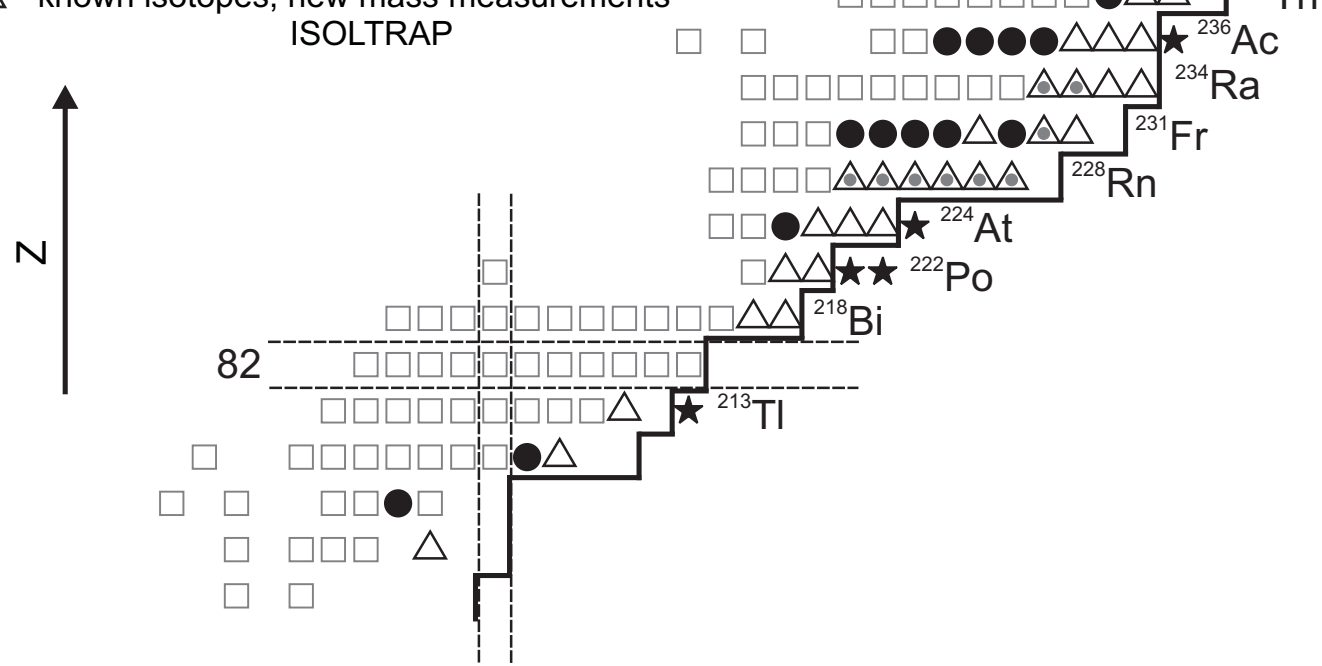

126

$\mathrm{N}$

Figure 5: Mass surface covered in this experiment. Nuclides, whose masses were measured for the first time [10], are indicated by open triangles. Since the publication of the doctoral thesis [10], which forms the basis of this work, new mass values for radon isotopes obtained from Penning trap experiments have been published [17]. The masses of three further nuclides, were measured at about the same time as in the SMS experiment [16]. Five new isotopes have been discovered in the element range from thallium to actinium [15]. The boundary of previously known neutron-rich isotopes is indicated by the solid line and was taken from the ENSDF database provided by the BNL-NNDC compilation [36] showing the status in April 2011. Meanwhile the border of known nuclei in this region of the nuclidic chart could be extended further [37]. The well-known masses indicated in the figure (open squares) have an average experimental error of about $8 \mathrm{keV}$ [30] and are used for calibration.

where $M$ is the mass of the neutral atom, $A$ its mass number and $u$ the atomic mass unit. 
Table 2: List of nuclei with the measured mass excess $(M E)$ values which have been improved in the present experiment [10]. The $\sigma_{M E}$ values are the corresponding errors.

\begin{tabular}{|r|r|r|r|r|r|}
\hline Element & $\mathrm{Z}$ & $\mathrm{N}$ & $\mathrm{A}$ & $\begin{array}{r}M E \\
(\mathrm{keV})\end{array}$ & $\begin{array}{r}\sigma_{M E} \\
(\mathrm{keV})\end{array}$ \\
\hline $\mathrm{Sn}$ & 50 & 78 & 128 & -83380 & 23 \\
$\mathrm{Sb}$ & 51 & 82 & 133 & -78899 & 23 \\
$\mathrm{Te}$ & 52 & 84 & 136 & -74468 & 23 \\
$\mathrm{La}$ & 57 & 87 & 144 & -74867 & 19 \\
$\mathrm{Ce}$ & 58 & 88 & 146 & -75610 & 37 \\
$\mathrm{Au}$ & 79 & 123 & 202 & -24353 & 23 \\
$\mathrm{Hg}$ & 80 & 127 & 207 & -16487 & 30 \\
$\mathrm{At}$ & 85 & 135 & 220 & 14376 & 14 \\
$\mathrm{Fr}$ & 87 & 137 & 224 & 21795 & 13 \\
& 87 & 138 & 225 & 23822 & 13 \\
& 87 & 139 & 226 & 27541 & 12 \\
& 87 & 140 & 227 & 29686 & 13 \\
& 87 & 142 & 229 & 35674 & 14 \\
$\mathrm{Ac}$ & 89 & 140 & 229 & 30690 & 12 \\
& 89 & 141 & 230 & 33838 & 16 \\
& 89 & 142 & 231 & 35763 & 13 \\
& 89 & 143 & 232 & 39154 & 13 \\
$\mathrm{Th}$ & 90 & 145 & 235 & 44018 & 13 \\
$\mathrm{~Pa}$ & 91 & 144 & 235 & 42289 & 14 \\
& 91 & 145 & 236 & 45334 & 14 \\
& 91 & 146 & 237 & 47528 & 13 \\
& 91 & 147 & 238 & 50894 & 16 \\
\hline
\end{tabular}

New accurate experimental data with the Penning trap system ISOLTRAP have been measured since our experiment was performed [17], which allow for a comparison of mass values from both methods. In Fig. 6 the values obtained by SMS and by ISOLTRAP $[16,17,18]$ are compared. The band around the mean value of the differences, $14 \mathrm{keV}$, reflects twice the rms value 
Table 3: List of nuclei with mass excess $(M E)$ values measured for the first time in the present experiment [10]. The $\sigma_{M E}$ values are the corresponding errors. Newly discovered isotopes are marked in bold.

\begin{tabular}{|r|r|r|r|r|r|}
\hline Element & $\mathrm{Z}$ & $\mathrm{N}$ & $\mathrm{A}$ & $\begin{array}{r}M E \\
(\mathrm{keV})\end{array}$ & $\begin{array}{r}\sigma_{M E} \\
(\mathrm{keV})\end{array}$ \\
\hline $\mathrm{Pt}$ & 78 & 124 & 202 & -22692 & 25 \\
$\mathrm{Tl}$ & $\mathbf{8 1}$ & $\mathbf{1 3 2}$ & $\mathbf{2 1 3}$ & $\mathbf{1 7 8 4}$ & $\mathbf{2 7}$ \\
$\mathrm{Bi}$ & 83 & 134 & 217 & 8730 & 18 \\
& 83 & 135 & 218 & 13216 & 27 \\
Po & 84 & 135 & 219 & 12681 & 16 \\
& 84 & 136 & 220 & 15263 & 18 \\
& $\mathbf{8 4}$ & $\mathbf{1 3 7}$ & $\mathbf{2 2 1}$ & $\mathbf{1 9 7 7 4}$ & $\mathbf{2 0}$ \\
& $\mathbf{8 4}$ & $\mathbf{1 3 8}$ & $\mathbf{2 2 2}$ & $\mathbf{2 2 4 8 6}$ & $\mathbf{4 0}$ \\
At & 85 & 136 & 221 & 16783 & 14 \\
& 85 & 137 & 222 & 20953 & 16 \\
& 85 & 138 & 223 & 23428 & 14 \\
& $\mathbf{8 5}$ & $\mathbf{1 3 9}$ & $\mathbf{2 2 4}$ & $\mathbf{2 7 7 1 1}$ & $\mathbf{2 2}$ \\
& 86 & 137 & 223 & 20381 & 12 \\
& 86 & 138 & 224 & 22453 & 13 \\
& 86 & 139 & 225 & 26526 & 13 \\
& 86 & 140 & 226 & 28753 & 14 \\
& 86 & 141 & 227 & 32905 & 23 \\
& 86 & 142 & 228 & 35234 & 29 \\
Fr & 87 & 141 & 228 & 33367 & 15 \\
& 87 & 143 & 230 & 39515 & 19 \\
& 87 & 144 & 231 & 42064 & 25 \\
Ra & 88 & 143 & 231 & 38212 & 14 \\
& 88 & 144 & 232 & 40496 & 14 \\
& 88 & 145 & 233 & 44322 & 16 \\
& 88 & 146 & 234 & 46893 & 31 \\
& 89 & 144 & 233 & 41308 & 13 \\
& 89 & 145 & 234 & 44841 & 14 \\
& 89 & 146 & 235 & 47357 & 14 \\
& $\mathbf{8 9}$ & $\mathbf{1 4 7}$ & $\mathbf{2 3 6}$ & $\mathbf{5 1 2 2 1}$ & $\mathbf{3 8}$ \\
& 90 & 146 & 236 & 46255 & 14 \\
& 90 & 147 & 237 & 49955 & 16 \\
\hline
\end{tabular}




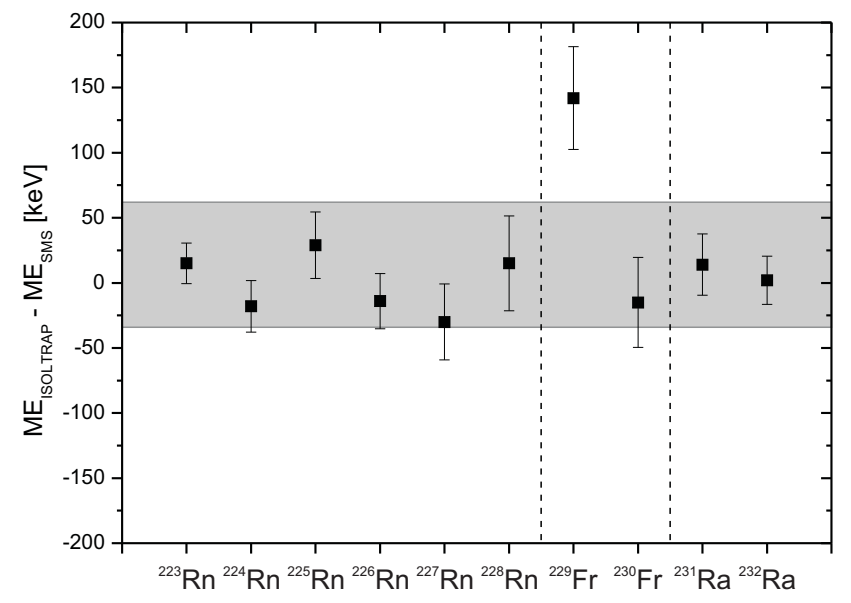

Figure 6: Comparison of the SMS mass values of the present work with the most recent results from ISOLTRAP for several Rn, Fr and Ra isotopes [16, 17, 18]. The half width of the grey band reflects the rms value of differences between both measurements and its center is the mean difference.

of differences $\sigma_{\text {rms }}=48 \mathrm{keV}$, which is calculated from

$$
\sigma_{r m s}=\sqrt{\frac{1}{n} \sum_{i=1}^{n}\left(M E_{\mathrm{SMS}}-M E_{\mathrm{ISOLTRAP}}\right)_{i}^{2}}
$$

where $n$ is the number of data points included and $M E_{\text {SMS }}$ and $M E_{\text {ISOLTRAP }}$ are the mass excess values obtained by SMS and ISOLTRAP, respectively.

This comparison demonstrates the excellent accuracy of both methods, which had already been observed in previous experiments [8]. One finds only a larger deviation for the nuclide ${ }^{229} \mathrm{Fr}$. Due to the larger mass excess value from the Penning-trap measurement compared to our value, one could conclude that an unknown isomeric state was actually measured in the earlier ISOLTRAP experiment [18]. However, the authors stated that ${ }^{229} \mathrm{Fr}$ could be stored in the precision trap only simultaneously with ${ }^{229} \mathrm{Ra}$. This could have led to the less accurate determination for the mass of ${ }^{229} \mathrm{Fr}$. Without the ${ }^{229} \mathrm{Fr}$ value the comparison is characterized by a better rms value of only $\sigma_{\mathrm{rms}}=19 \mathrm{keV}$.

For those nuclides, for which a mass value has been measured for the first time in this work, a comparison can be made with the extrapolated values given in the AME2003. Likewise, mass values of nuclides, for which 

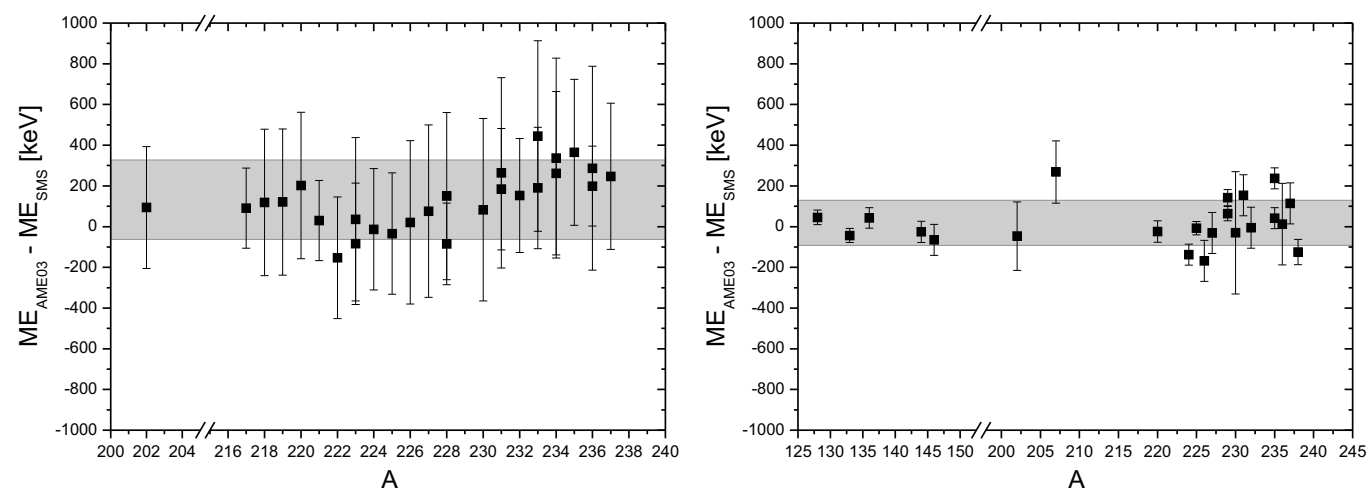

Figure 7: Mass values from this work compared to the values of AME2003 compilation. The half-width of the grey band reflects the rms value of the differences and its center is the mean difference. Left panel: Comparison of the new experimental masses with the extrapolated values of the AME2003. Right panel: Comparison of previously known masses which have been improved by the present experiment.

the uncertainties have been reduced, can be compared with the previous values contained in the AME2003. This comparison is shown in Fig. 7. The mean deviation between the new mass values and the AME2003 predictions is $133 \mathrm{keV}$ with an rms value of differences of $195 \mathrm{keV}$. The mean deviation for the improved mass values is $19 \mathrm{keV}$ with an rms deviation of $111 \mathrm{keV}$.

Systematic effects in the mass surfaces can often most easily be found from an investigation of mass differences or even multifold mass differences.

For the element range covered in the present experiment, the two-neutron separation energies $\left(S_{2 n}\right)$ values are shown in Fig. 8. They are defined by

$$
S_{2 n}=-M E(A, Z)+M E(A-2, Z)+2 \cdot M E(1,0),
$$

where $M E(A, Z), M E(A-2, Z)$, and $M E(1,0)$ are the mass excess values of the ion of interest, of the two-neutron more deficient isotope of the same element, and of the neutron, respectively.

The $\mathrm{S}_{2 n}$ values have a smooth trend as a function of the neutron number in general except at the shell closure at $N=126$. Similar nuclear structure information can be directly observed with $\delta \mathrm{V}_{p n}$ values [38]. For even-even nuclides, the $\delta \mathrm{V}_{p n}$ value is defined by

$$
\delta V_{p n}^{e e}(Z, N)=-\frac{1}{4}\left[S_{2 n}(Z, N)-S_{2 n}(Z-2, N)\right]
$$



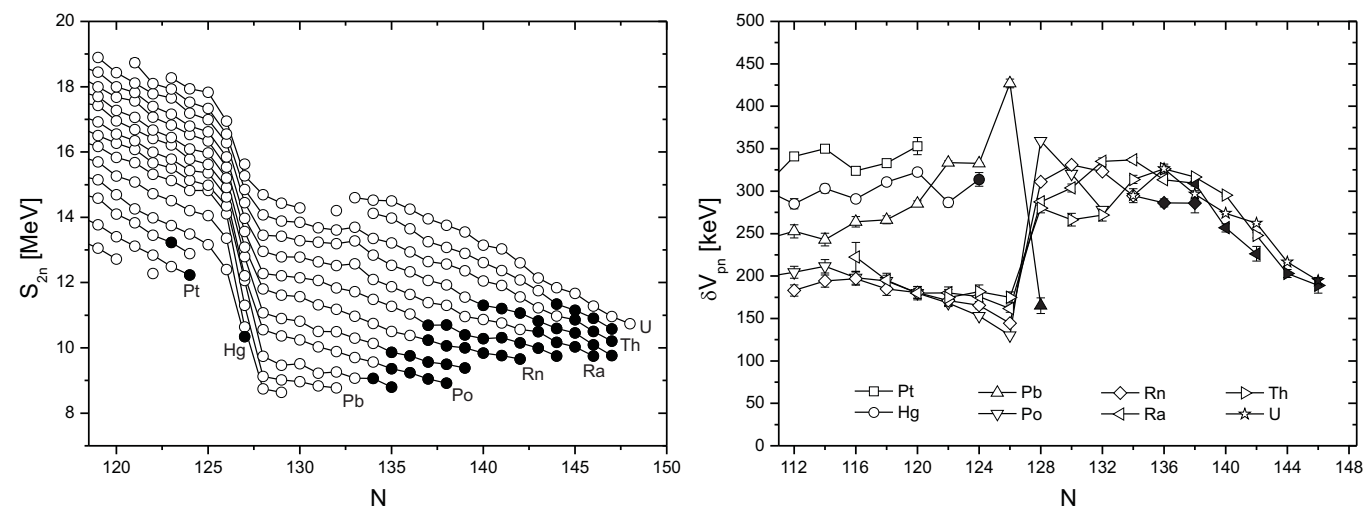

Figure 8: Panel on the left hand side: Experimental two-neutron separation energies. The new values of this experiment are indicated with filled circles the other data are taken from ref. [30]. Panel on the right hand side: The shell closure at $\mathrm{N}=126$ is clearly demonstrated in the proton-neutron interaction represented by the $\delta \mathrm{V}_{p n}$ values. The full symbols indicate the new values obtained from the present mass excess data. The value for ${ }^{210} \mathrm{~Pb}$ was derived using the value for ${ }^{208} \mathrm{Hg}$ published in $[10,14]$. The open symbols represent the data from ref. [30].

The $\delta \mathrm{V}_{p n}$ values can yield information on the average interaction of the protons and neutrons in their shell occupancy [14, 38].

Indeed, they are double differences of the binding energies and can reflect, in a simplified shell-model picture, an averaged proton-neutron interaction and thus can indicate shell and sub-shell closures. This interaction is depicted in Fig. 8 for even-even isotopes as a function of the neutron number. The shell closure at $\mathrm{N}=126$ is clearly manifested and possible structural changes as suggested in reference [17] are confirmed. The value for ${ }^{210} \mathrm{~Pb}$ was calculated using the mass excess value for ${ }^{208} \mathrm{Hg}$ published in $[10,14]$. As stated above, both analysis methods are in very good agreement.

Nuclear mass models have primarily two goals: they must reproduce and explain the available experimental data, and they have to predict values for the unknown territory, i.e., in our case to extend the information towards the possible path of the r-process [39, 40]. Here, we show a representative comparison of our new experimental mass values to the widely-used and most accurate macroscopic-microscopic model FRDM [41], the semiempirical complex mass formula of Duflo and Zuker [42], and two microscopic Hartree-Fock-Bogoliubov theories, namely HFB-14 [43] and HFB-17 [44].

The experimental results are compared with the theoretical predictions 

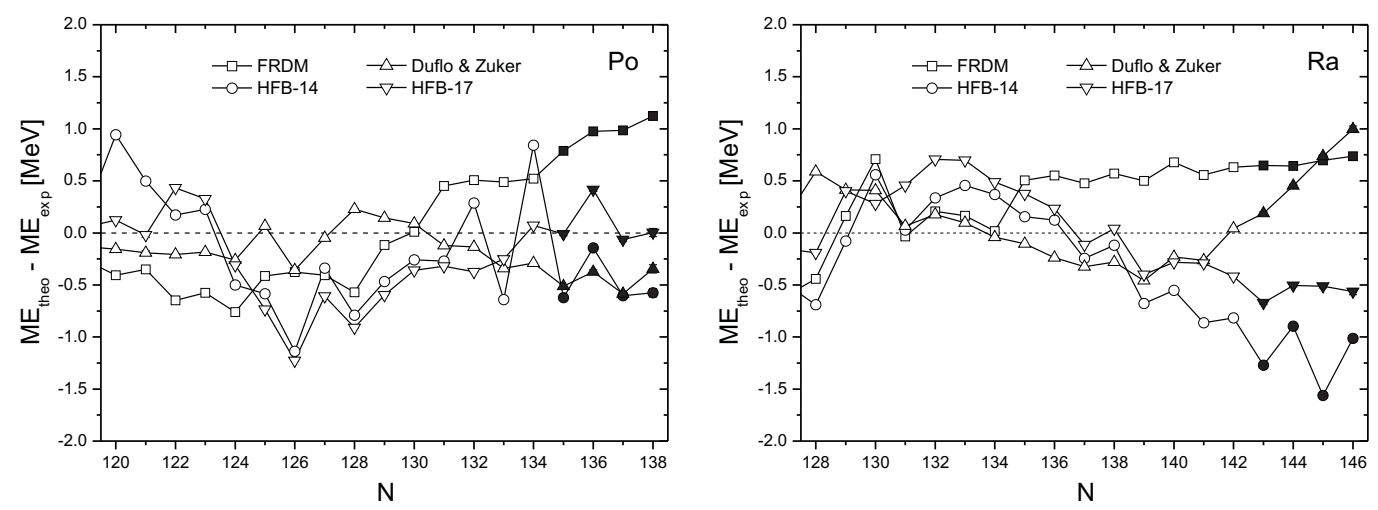

Figure 9: Comparison of experimental Mass Excess $(M E)$ data for Po and Ra isotopes with different theoretical models [41], [42], [43], [44]. The experimental data are taken from ref. [30] and the present experiment indicated by full symbols.

for Polonium and Radium isotopes. The comparison in Fig. 9 clearly shows the deficiency of the models. The deviations from the experimental data systematically increase with increasing neutron number, i.e., with the distance from the previous experimentally known mass values.

The rms-deviations of the newly measured $M E$ values, marked with filled symbols in figure 9 calculated with the different mass models for Po isotopes are $976 \mathrm{keV}, 464 \mathrm{keV}, 525 \mathrm{keV}, 211 \mathrm{keV}$ corresponding to the references [41], [42], [43], [44], respectively. The corresponding rms-deviations for Ra are 683 $\mathrm{keV}, 667 \mathrm{keV}, 1213 \mathrm{keV}, 567 \mathrm{keV}$ related for the references [41], [42], [43], [44], respectively.

Besides the generally valid observation of the stronger deviation for the more n-rich isotopes, the odd-even fluctuation in the comparison reveals another shortcoming in the theoretical models. These statements also hold for other elements within the mass area mapped in our experiment. The most crucial test is the direct comparison of the experimental and theoretical binding energies, because when binding energy differences are compared, systematic problems could partially cancel. In Fig. 10 the binding energy differences in form of $\mathrm{S}_{2 n}$ values for Po and Ra isotopes are again compared. The rms-deviations of the $\mathrm{S}_{2 n}$ values for the different mass models for Po isotopes, where our new mass excess values contribute, are $299 \mathrm{keV}, 103 \mathrm{keV}$, $538 \mathrm{keV}, 296 \mathrm{keV}$ corresponding to the references [41], [42], [43], [44], respectively. The corresponding rms-deviations for Ra are $70 \mathrm{keV}, 492 \mathrm{keV}$, 

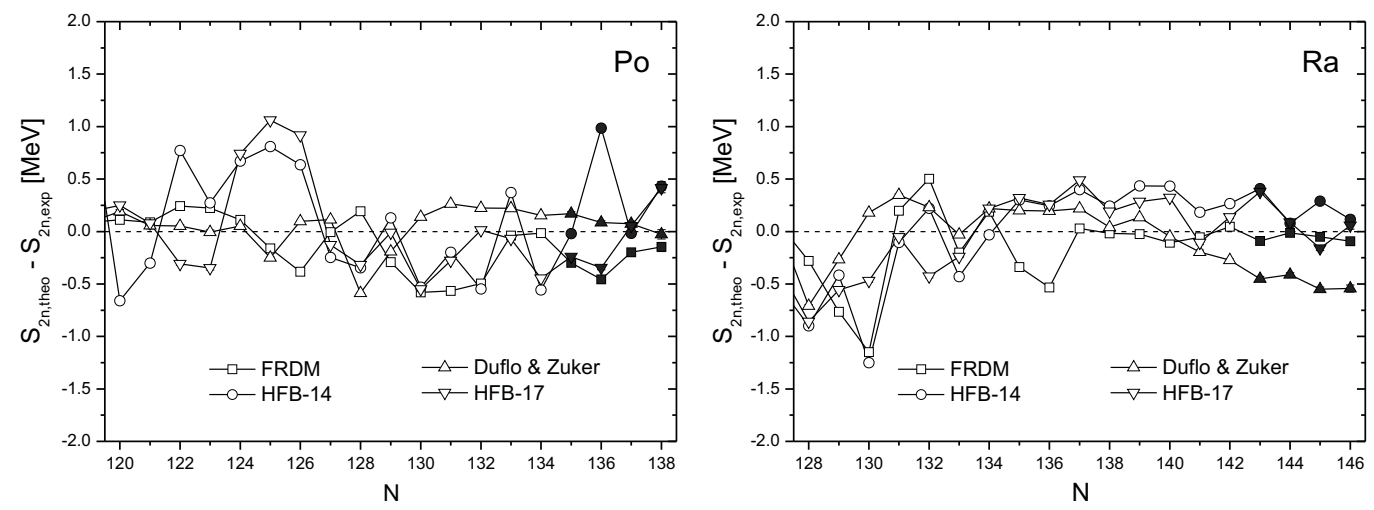

Figure 10: Comparison of experimental $S_{2 n}$ - values for Po and Ra isotopes with different theoretical models [41], [42], [43], [44]. The experimental data are taken from ref. [30] and the present experiment indicated by full symbols.

$260 \mathrm{keV}, 212 \mathrm{keV}$ related to the references [41], [42], [43], [44], respectively. At the first glance one can observe that the deviations are really in general smaller because in this way the systematic inherent deficiency partially cancels due to the fact that one only judges differences for each model.

\section{Summary}

The presented masses of exotic nuclei measured at the FRS-ESR experimental facility contribute significantly to the present knowledge in the heavy element range between $\mathrm{Pt}$ and $\mathrm{U}$ for neutron-rich isotopes. For the elements Ac, At, Po, and Tl, the most neutron-rich isotopes have been discovered in the present experiment [15]. Meanwhile, these nuclei have also been observed in other FRS experiments with different scientific goals. The importance of cold fragmentation and nuclear charge-changing reactions is manifested by the observation and investigation of the so far most neutron-rich isotopes in the region of lead and uranium. Time resolved Schottky spectrometry was applied to measure new masses with an average accuracy of about $19 \mathrm{keV}$.

The observation of a systematic dependence for the recalculated reference masses by the matrix evaluation led to the conclusion that the radial velocity profile of the electron beam in the cooler has to be taken into account. Our representative illustration of the physical impact of these experimental results demonstrates the shortcomings of the present theoretical description, 
especially for the new masses and the discovered isotopes. In such timeresolved SMS experiments one also obtains new information on the decay properties of the isotopes, including isomers, covered in the measured frequency spectra, e.g. ref. $[45,46]$. The results on new isomeric states and lifetimes will be discussed in a forthcoming publication.

In the near future, the intensity upgrade program for the GSI accelerators will substantially contribute to improve the accuracy of the spectroscopy information for the most exotic isotopes and will also give access to more unknown nuclides. In particular, we aim at measurements near and at the $\mathrm{N}=126$ shell closure which is very relevant for the astrophysical models describing the r-process paths.

\section{Acknowledgement}

It is a pleasure to thank the technical staffs of the accelerators, the FRS, and the target laboratory for their valuable contribution to the beam quality and experimental setups. The authors gratefully acknowledge fruitful discussions with K. Blaum, I. Borzov, and R.F. Casten. We thank very much the Helmholtz Association (HGF) for the support of the Helmholtz University Young Investigators Group VH-NG-033, which was a basis for the strong collaboration of the University of Gießen and the research center GSI. One of us, P.M. W., would like to acknowledge the financial support from the UK STFC and AWE plc. B. S., is partially supported by NECT and NSFC 11105010. Two of us, K. S.I. and Z. P. would like to acknowledge the financial support by Narodowe Centrum Nauki (Poland) grant No. 2011/01/B/ST2/05131.

\section{References}

[1] K. Otsuki at al., New Astronomy 8 (2003) 767.

[2] J.J. Cowan, C. Sneden, Nature 440 (2006) 1151.

[3] Z. Patyk et al., Nucl. Phys. A 491 (1989) 267.

[4] J. Kurcewicz, S. Pietri et al., GSI Experimental Proposal S392 (2009).

[5] H. Geissel et al., Nucl. Instr. Meth. B 70 (1992) 286.

[6] B. Franzke, Nucl. Instr. Meth. B 24-25 (1987) 18. 
[7] B. Franzke, H. Geissel, G. Münzenberg, Mass Spectrom. Rev. 27(5) (2008) 428 .

[8] Yu.A. Litvinov et al., Nucl. Phys. A 756 (2005) 3.

[9] W.R. Plaß, Hyperfine Interact. 173 (2006) 19.

[10] L. Chen, doctoral thesis, Justus-Liebig-University Gießen (2008).

[11] H. Geissel et al., AIP Conf. Proceed. 831 (2006) 108.

[12] F. Bosch et al., Int. J. Mass spectrom. 251 (2006) 212.

[13] H. Geissel et al., Eur. Phys. J. Special Topics 150 (2007) 109.

[14] L. Chen et al., Phys. Rev. Lett. 102 (2009) 122503.

[15] L. Chen et al., Phys. Lett. B 691 (2010) 234.

[16] F. Herfurth et al., Eur. Phys. J. A 25 (2005) 17.

[17] D. Neidherr et al., Phys. Rev. Lett. 102 (2009) 112501.

[18] C. Weber et al., Nucl. Phys. A 803 (2008) 1.

[19] G. Bollen, Lect. Notes Phys. 651 (2004) 169.

[20] D. Lunney, J. M. Pearson and C. Thibault, Rev. Mod. Phys. 75 (2003) 1021.

[21] K. Blaum, Phys. Rep. 425 (2006) 1.

[22] J. Benlliure et al., Eur. Phys. J. Special Topics 150 (2007) 309.

[23] H. Alvarez-Pol et al., Phys. Rev. C 82 (2010) 041602(R).

[24] J. Kurcewicz et al., GSI Annual Report 2010 (2011) 144.

[25] K. Blasche, B. Franczak, in: Proc.: 3rd Eur. Part. Acc. Conf., Berlin, (1992) 9.

[26] M. Steck, Nucl. Instr. Meth. A 532 (2004) 357.

[27] T. Radon et al., Nucl. Phys. A 677 (2000) 75. 
[28] H. Poth, Phys. Rep. 196 (1990) 135.

[29] R. W. Hasse, Phys. Rev. Lett. 86 (2001) 3028.

[30] A. H. Wapstra, G. Audi, C. Thibault, Nucl. Phy. A 729 (2003) 129.

[31] P. Möller et al., At. Data Nucl. Data Tables 59 (1995) 185.

[32] G.C. Rodrigues et al., At. Data Nucl. Data Tables 86 (2004) 117.

[33] M. Steck private communication 2011.

[34] J. Benlliure et al., Nucl. Phys. A 660 (1999) 87.

[35] A. Kelić et al., Phys. Rev. C 70 (2004) 064608.

[36] http://www.nndc.bnl.gov/; http://www.nndc.bnl.gov/ensdf/

[37] H. Alvarez-Pol et al., Phys. Rev. C 82 (2010) 041602(R).

[38] D.S. Brenner, R. B. Cakirli, R. F. Casten, Phys. Rev. C 73 (2006) 034315.

[39] J. J. Cowan, F.-K. Thielemann, Phys. Today 57 (2004) 47.

[40] B. Sun et al., Phys. Rev. C 78 (2008) 025806.

[41] P. Möller et al., At. Data Nucl. Data Tables 59 (1995) 185.

[42] J. Duflo, A.P. Zuker, Phys. Rev. C 52 (1995) R23.

[43] http://www-astro.ulb.ac.be/Nucdata/Masses/hfb14-plain; S. Goriely, M. Samyn, J.M. Pearson, Phys. Rev. C 75 (2007) 064312 and references therein.

[44] S. Goriely et al., Eur. Phys. J. A 42 (2009) 547; S. Goriely et al., Phys. Rev. Lett. 102 (2009) 152503.

[45] Yu. A. Litvinov et al., Phys. Rev. Lett. 99 (2007) 262501.

[46] M.W. Reed et al., Phys. Rev. Lett. 105 (2010) 172501. 